\title{
A description of clinician reported diagnosis of type 2 diabetes and other non-type 1 diabetes included in a large international multicentered pediatric diabetes registry (SWEET)
}

\author{
Danièle Pacaud MD, FRCPC ${ }^{1}$ | Anke Schwandt $\mathrm{MSc}^{2,3}$ | Carine de Beaufort MD, $\mathrm{PhD}^{4,5}$ | \\ Kristina Casteels MD, $\mathrm{PhD}^{6,7}$ | Jacques Beltrand $\mathrm{MD}, \mathrm{PhD}^{8,9}$ | \\ Niels H. Birkebaek MD, PhD ${ }^{10}$ | Myrna Campagnoli MD ${ }^{11}$ | Natasa Bratina MD, PhD ${ }^{12}$ | \\ Catarina Limbert MD ${ }^{13}$ | Stephen MP O'Riordan MD ${ }^{14}$ | Rogério Ribeiro MD, PhD ${ }^{15}$ | \\ Andriani Gerasimidi-Vazeou MD ${ }^{16}$ | Lenka Petruzelkova MD ${ }^{17}$ |
} Rasa Verkauskiene MD, PhD ${ }^{18}$ | Iveta Dzivite Krisane MD, PhD ${ }^{19}$ | the SWEET Study Group

\author{
${ }^{1}$ Alberta Children's Hospital Research Institute, \\ University of Calgary, Calgary, Canada \\ ${ }^{2}$ Institute of Epidemiology and Medical Biometry, \\ ZIBMT, University of Ulm, Ulm, Germany \\ ${ }^{3}$ German Center for Diabetes Research (DZD), \\ Munich-Neuherberg, Germany \\ ${ }^{4}$ Diabetes and Endocrinology Care Clinique \\ Pédiatrique (DECCP), Centre Hospitalier de \\ Luxembourg, Luxembourg, Luxembourg \\ ${ }^{5}$ Division of Paediatric Endocrinology, \\ University Hospital Brussels, Brussels, Belgium \\ ${ }^{6}$ Department of Pediatrics, University \\ Hospitals Leuven, Leuven, Belgium \\ ${ }^{7}$ Department of Development and \\ Regeneration, KU Leuven, Leuven, Belgium \\ ${ }^{8}$ Service d'endocrinologie gynécologie et \\ diabétologie pédiatrique, Hôpital Universitaire \\ Necker Enfants Malades, Assistance publique \\ Hôpitaux de Paris, Paris, France \\ ${ }^{9}$ Faculté de médecine Paris Descartes, \\ Université Sorbonne Paris cité, Paris, France \\ ${ }^{10}$ Department of Pediatrics, Aarhus University \\ Hospital, Aarhus, Denmark \\ ${ }^{11}$ Centro de Diabetes de Curitiba, Paraná, Brazil \\ ${ }^{12}$ Departement of endocrinology, diabetes and \\ metabolic diseases, University Childrens hospital, \\ University medical centre, Ljubljana, Slovenia \\ ${ }^{13}$ Hospital Dona Estefânia, Unit of Pediatric \\ Endocrinology and Diabetes, Lisbon, Portugal \\ ${ }^{14}$ Paediatric Endocrinology, Department of \\ Paediatrics \& Child Health, Cork University \\ Hospital, Cork, Ireland \\ ${ }^{15}$ Associação Protectora dos Diabéticos de \\ Portugal (APDP), Lisbon, Portugal \\ ${ }^{16}$ Department of Pediatrics, P \& A Kyriakou \\ Children's Hospital, Diabetes Centre, Athens, \\ Greece
}

Background: Although type 1 diabetes (T1D) remains the most frequent form of diabetes in individuals aged less than 20 years at onset, other forms of diabetes are being increasingly recognized.

Objectives: To describe the population of children with other forms of diabetes (non-type 1) included in the multinational SWEET (Better control in Pediatric and Adolescent diabeteS: Working to crEate CEnTers of Reference) database for children with diabetes.

Methods: Cases entered in the SWEET database are identified by their physician as T1D, type 2 diabetes (T2D) and other types of diabetes according to the ISPAD classification. Etiologic subgroups are provided for other types of diabetes. Descriptive analyses were tabulated for age at onset, gender, daily insulin doses, and hemoglobin A1c (A1C) for each type and subtype of diabetes and when possible, values were compared.

Results: Of the 27104 patients included in this report, 95.5\% have T1D, 1.3\% T2D, and 3.2\% other forms of diabetes. The two most frequent etiologies for other forms of diabetes were maturity onset diabetes of the young (MODY) $(n=351)$ and cystic fibrosis-related diabetes (CFRD) $(n=193)$. The cause was unknown or unreported in $10 \%$ of other forms of diabetes. Compared with T1D, children with T2D and CFRD were diagnosed at an older age, took less insulin and had lower A1C (all $P<.0001$ ).

Conclusion: In centers included in SWEET, forms of diabetes other than type 1 remain rare and at times difficult to characterize. Sharing clinical information and outcome between SWEET centers on those rare forms of diabetes has the potential to improve management and outcome.

KEYWORDS

MODY, monogenic diabetes, neonatal diabetes, pediatric diabetes, type 2 diabetes 
${ }^{17}$ Department of Paediatrics, University Hospital Motol and 2nd Faculty of Medicine, Charles University in Prague, Prague, Czech Republic

${ }^{18}$ Institute of Endocrinology, Lithuanian University of Health Sciences, Medical Academy, Kaunas, Lithuania

\section{1 | INTRODUCTION}

Although type 1 diabetes (T1D) remains the most frequent form of diabetes in individuals aged less than 20 years at onset, other forms of diabetes including type 2 diabetes (T2D) and monogenic forms of diabetes are being increasingly recognized. ${ }^{1}$ Reports from different sources suggest that between $2 \%$ and $15 \%$ of children affected by diabetes will have non-T1D. ${ }^{2,3}$ Although T2D is being recognized more frequently and could represent up to $11 \%$ of children affected by diabetes in the USA, ${ }^{4}$ it remains a fairly rare form of diabetes in other countries. Prevalence of T2D is often reported as being similar or even lower than monogenic forms of diabetes. ${ }^{5}$

The management of diabetes in children is changing rapidly and more options are becoming available. Management of T1D is an intensive process requiring significant education, time, and resources. However, this management approach may not be required in other forms of diabetes such as in glucokinase (GCK) gene mutations (MODY 2) where use of insulin exposes the child to unnecessary daily injections and risks of hypoglycemia. ${ }^{6}$ Making the correct diagnosis of diabetes type and subtype is important to offer the most appropriate treatment, to conduct targeted screening of complications and associated conditions, and for genetic counseling of the families. ${ }^{3}$

SWEET comprises a large multinational consortium of pediatric diabetes clinics collecting basic diabetes-related information on their patients in a single, standardized database. This provides a unique opportunity to evaluate the frequency, presentation, treatment, and follow up of forms of clinician reported diagnosis of diabetes other than type 1. Hence, the aim of this manuscript is to describe the population of children with other forms of diabetes (non-type 1) included in the multinational SWEET database for children with diabetes.

\section{2 | METHODS}

SWEET is the acronym for "Better control in Pediatric and Adolescent diabeteS: Working to crEate CEnTers of Reference," a multinational initiative to improve diabetes care and outcomes in youth with diabetes. Before being allowed to join, each center has to meet specific entry criteria demonstrating their pediatric diabetes expertise and compliance with the International Society for Pediatric and Adolescent Diabetes clinical practice guidelines. ${ }^{7}$ For the data collection, the SWEET project incorporates data from heterogeneous sources: centers may use DPV ("Diabetes-Patienten-Verlaufskodumentation," https://sweet.zibmt.uni-ulm.de/software.php), DIAMAX, download data of existing registries or use own local databases to collect data. Since 2006, twice per year, prospective data extracted from clinical visit documentation are transferred from individual centers to the SWEET data management unit at the Institute of Epidemiology and Medical Biometry, University of Ulm, Ulm, Germany. Data are
${ }^{19}$ Children's University Hospital Children's Endocrinology Centre, Riga Stradins University, Riga, Latvia

Corresponding Author: Danièle Pacaud, MD, FRCPC, Alberta Children's Hospital, University of Calgary, 2888 Shaganappi Trail NW, Calgary, Alberta T3B 6A8, Canada (daniele.pacaud@ahs.ca).

examined for inconsistency or improbability and reported back to centers for verification. Moreover, reports are generated comparing outcomes between participating centers.

Individual centers obtained local ethics approval. Individual informed consent from patient or parents in the case of minors were obtained when required by local regulations.

As of February 2016, SWEET included 48 participating centers (37 from Europe) with 281906 visits in 28713 patients. After excluding patients with missing gender, age, diabetes duration or diabetes type, diagnosed after the age of $20 \mathrm{y}$, and patients with glucose intolerance not yet diagnosed with diabetes, 27104 patients remained for analysis (Figure 1). For each patient, the data from the latest visit available was used for categorical variables including diabetes type and subtype. Data from continuous variables were aggregated from visits of the last 2 years of observation so that individual patients are represented only once.

This is a multicentered cross-sectional observational descriptive study. Type and subtypes of diabetes are determined by their treating physician and reported in the database according to the ISPAD classification table ${ }^{8}$ (see our Table 1). At this time, the SWEET database does not include information on the method used to make the diagnosis or on specific mutations identified. Types of diabetes reported are type 1 , type 2 , gestational diabetes or other forms of diabetes. If a child was diagnosed with diabetes before the age of 6 months, but no specific genetic diagnosis was mentioned, this case was included under genetic defect of $\beta$-cell function. For descriptive purposes, reported diabetes subtypes were regrouped into genetic defect of $\beta$-cell function (MODY and neonatal diabetes), genetic defects of insulin action, cystic fibrosis-related diabetes (CFRD), other disease of the exocrine pancreas (excluding CFRD), endocrinopathies, drug or

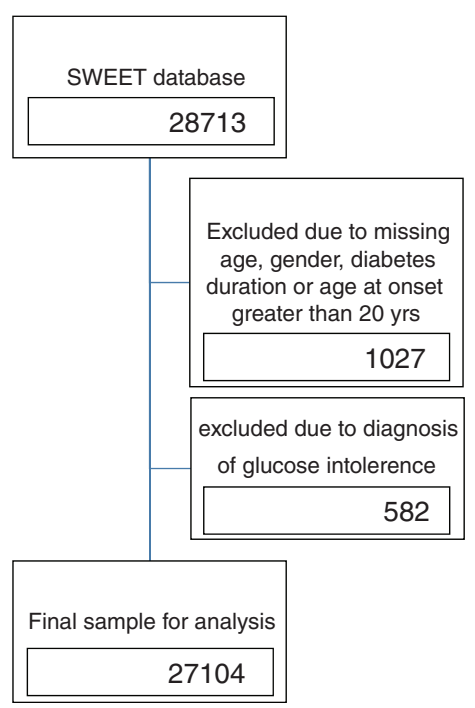

FIGURE 1 Sample description. 


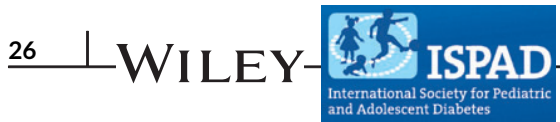

TABLE 1 Number of cases of diagnosis subtype categories for other forms of diabetes observed in the SWEET database

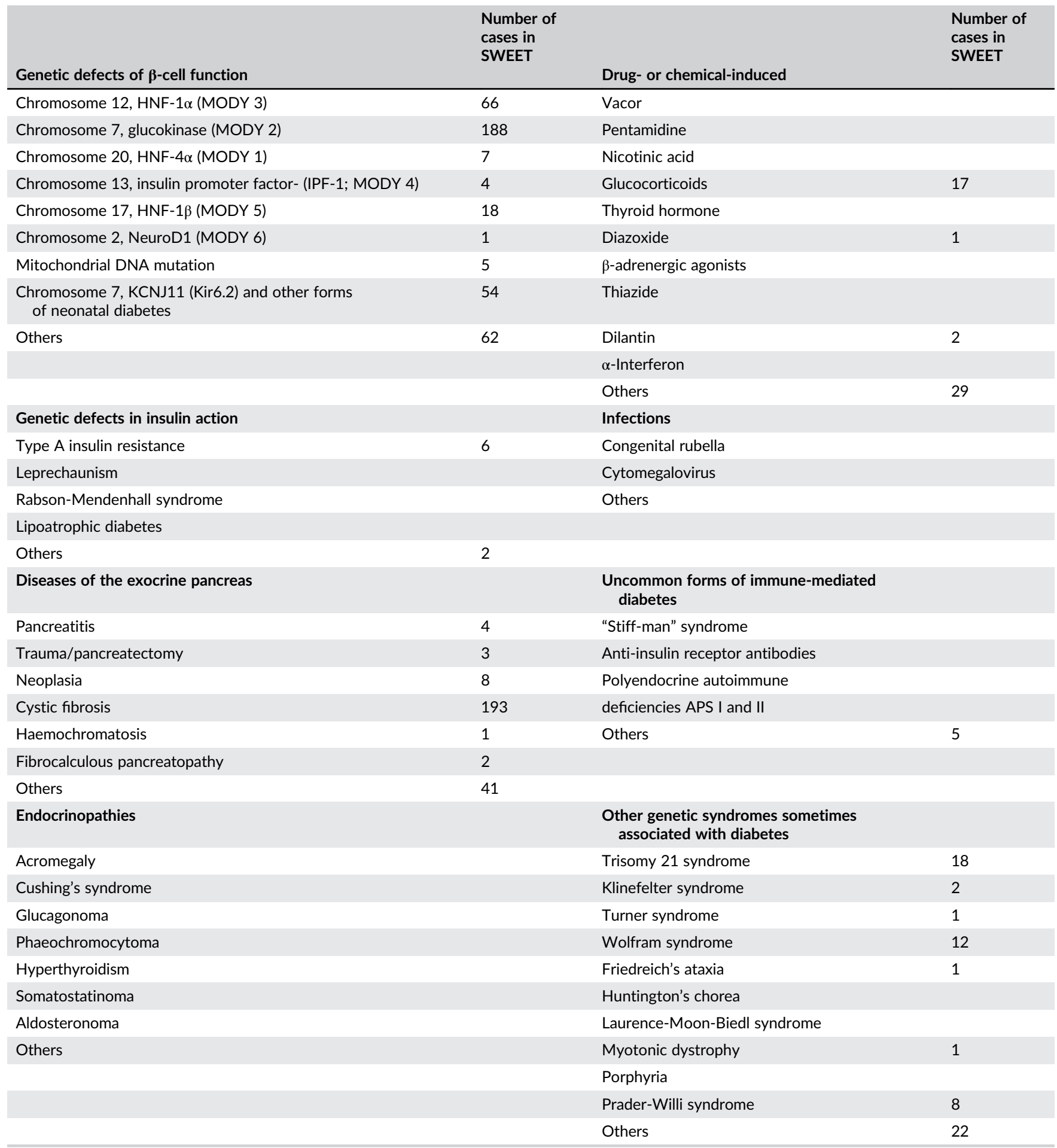

chemically induced diabetes, infections, uncommon forms of immune-mediated diabetes and other genetic syndromes sometimes associated with diabetes.

The main clinical information available for this paper include age at onset (y), gender, type, and subtype of diabetes and for each visit height $(\mathrm{cm})$, weight $(\mathrm{kg})$, body mass index $\left(\mathrm{BMI}, \mathrm{kg} / \mathrm{m}^{2}\right)$, BMI $\mathrm{z}$ scores [according to the World Health Organization (WHO) growth charts], ${ }^{9}$ hemoglobin $\mathrm{A} 1 \mathrm{c}$ (A1C, \%), use of insulin (yes/no), total daily insulin dose (units per $\mathrm{kg}$ ) and use of oral antihyperglycemic agents (yes/no). In order to adjust for differences between laboratories for
A1C measurements, multiple of the mean (MOM) method ${ }^{10}$ was used to mathematically standardize $\mathrm{A} 1 \mathrm{C}$ values to the reference range of the Diabetes Control and Complications Trial (DCCT, 4.0\%-6.0\%; 20-42 $\mathrm{mmol} / \mathrm{mol}$ ).

In preparation for this special supplement on SWEET, we asked all participating centers to complete an online survey including questions on where would children and adolescents with cystic fibrosisrelated diabetes, T2D, and adolescents with diabetes in pregnancy be followed. Of 48 centers 32 completed the survey (response rate $67 \%$ ). 


\section{1 | Statistical analysis}

Results are presented as median with lower and upper quartile for continuous variables or as number and percentage for binary or categorical variables. Wilcoxon test was used to compare continuous variables between groups and chi-square test for categorical variables. Linear regression models compared the metabolic control (A1C) between T1D, T2D, and CFRD adjusted for age, diabetes duration, and gender were presented as adjusted mean and standard error (SE). A Spearman correlation was used to assess the association between center size and percentage of rare diabetes or T2D from each center.

All analyses were done using Statistical Analysis Software 9.4 (SAS, SAS Institute Inc., Cary, NC, USA). Two-sided $P$-value $<.05$ was considered as significant.

\section{3 | RESULTS}

As shown in Figure 2, of the 27104 patients included in this report, 25889 (95.5\%) had T1D, 347 (1.3\%) T2D, 2 (<0.01\%) gestational diabetes, and 866 (3.2\%) other forms of diabetes. For those identified by their physician as having another form of diabetes, a specific subclass was provided in $90 \%$ and unknown or unreported in $10.0 \%$. The distribution of other forms of diabetes can be seen in Figure 2. The most frequent groups were MODY $2(n=188)$ and CFRD $(n=193)$.

The frequency of the specific etiologies of diabetes can be seen in Table 1. The clinical characteristics of these different subgroups can be found in Table 2. Of note, a total of 351 children were categorized as MODY (total of MODY 1, 2, 3,4,5,6). Although no

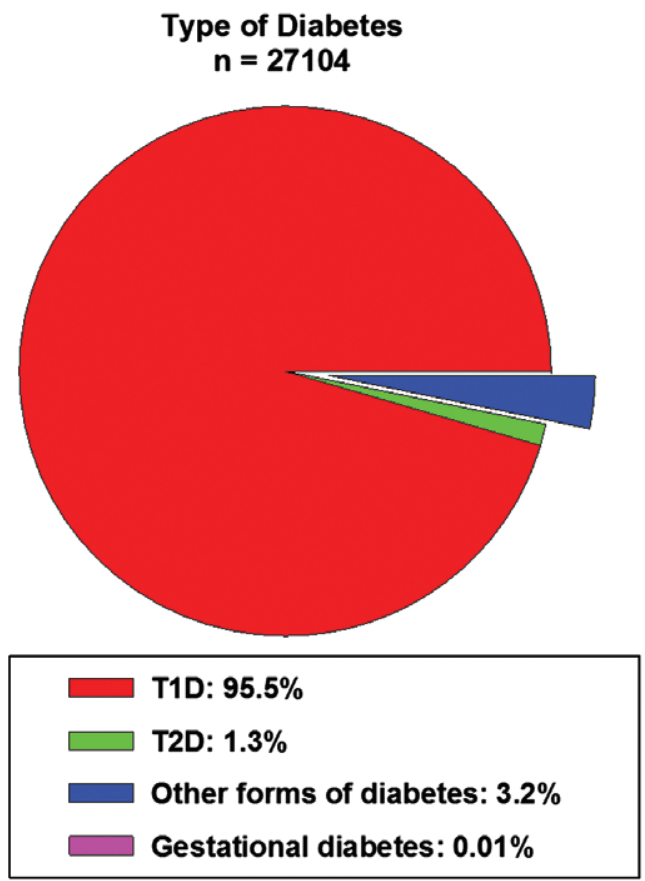

statistical analysis was performed due to the small group size, it appears that children with MODY 2 are younger at diagnosis. Only $7 \%$ were on insulin with a median insulin dose of $0.2(0.1 ; 0.5) \mathrm{U} / \mathrm{kg} /$ d. However, in this dataset, the reported A1C levels appear similar in all MODY groups. In addition, Table 2 presents the characteristics of children with diabetes because of monogenic forms of neonatal diabetes, disease of the exocrine pancreas (excluding CFRD), drug or chemical or associated with a genetic syndrome.

In unadjusted comparison between children with T1D and with $\mathrm{T} 2 \mathrm{D}$, children and adolescents with T2D were older, more frequently girls, and older at diagnosis (Table 3). They also have a shorter duration of diabetes, lower $\mathrm{A} 1 \mathrm{C}$, lower daily insulin doses per kilogram, and higher BMI z-scores. In contrast, children with T2D and CFRD had similar duration of diabetes, yet shorter than for those with T1D. T2D and CFRD also had similar female predominance in contrast with T1D that showed no gender difference. Children with CFRD had the lowest BMI z-score. When adjusting for age, gender, and duration of diabetes, $\mathrm{A} 1 \mathrm{C}$ remained different between groups (T1D $8.21 \pm 0.01 \%$ or $66 \pm 0.1 \mathrm{mmol} / \mathrm{mol}$ vs. T2D $7.68 \pm 0.10 \%$ or $61 \pm 1 \mathrm{mmol} / \mathrm{mol}, \mathrm{p}<0.0001)$ and children with CFRD were found to have the lowest A1C compared to both T1D (CFRD $6.32 \pm 0.12 \%$ or $45 \pm 1 \mathrm{mmol} / \mathrm{mol}$ vs. T1D $8.21 \pm 0.01 \%$ or $66 \pm$ $0.1 \mathrm{mmol} / \mathrm{mol}, \mathrm{p}<0.0001$ ) and T2D (CFRD $6.35 \pm 0.15 \%$ or $46 \pm 2$ $\mathrm{mmol} / \mathrm{mol}$ vs. T2D $7.75 \pm 0.12 \%$ or $62 \pm 1 \mathrm{mmol} / \mathrm{mol}, \mathrm{p}<0.0001$ ).

Of the 48 diabetes clinics participating, 7 reported on more than 1000 patients, 33 between 150 to 1000 patients, and 8 on less than 150 patients since the beginning of their contribution to SWEET. The proportion of $\mathrm{T} 2 \mathrm{D}$ ranged from $0 \%$ to $8.3 \%$. There was a weak correlation between center size and the percentage of patients with T2D per center (Spearman Rho 0.45, $P=.001$ ). The proportion of other forms

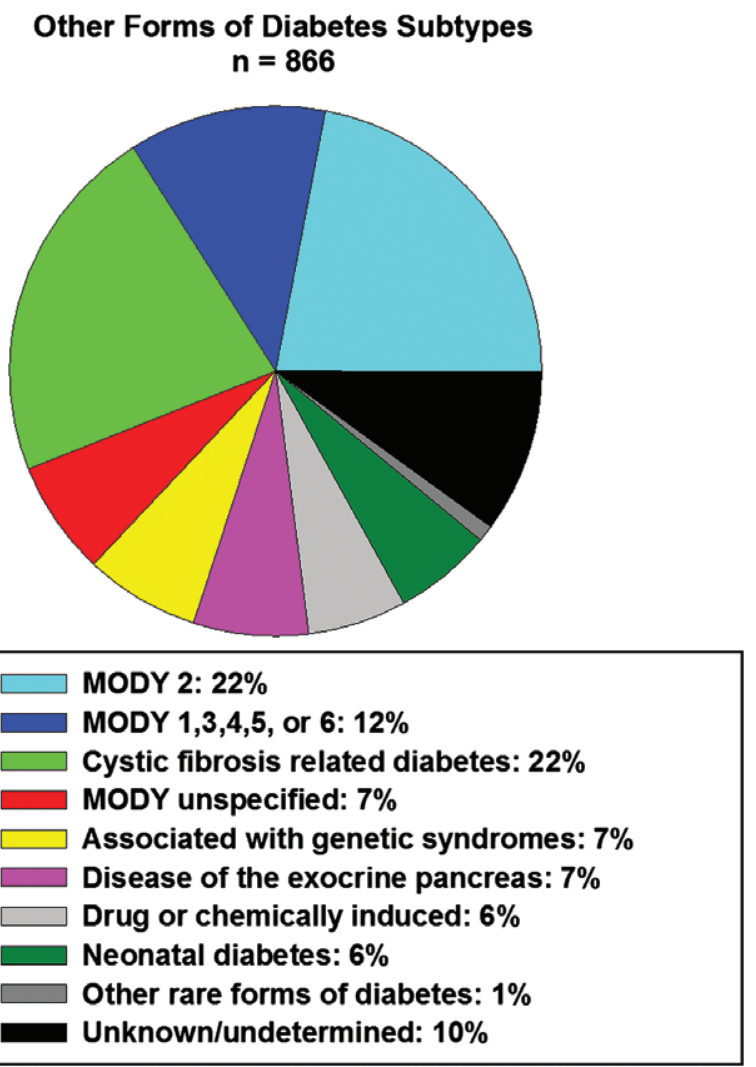




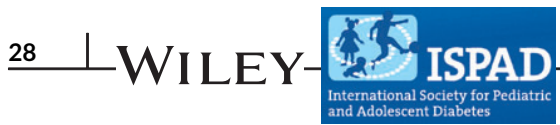

TABLE 2 Clinical characteristics of rarer forms of diabetes ${ }^{1,2}$

\begin{tabular}{|c|c|c|c|c|c|c|c|}
\hline & $\begin{array}{l}\text { MODY } 2 \\
(n=188)\end{array}$ & $\begin{array}{l}\text { MODY } 1,3 \\
4,5 \\
6(n=101)\end{array}$ & $\begin{array}{l}\text { MODY } \\
\text { unspecified or } \\
\text { undetermined } \\
(n=62)\end{array}$ & $\begin{array}{l}\text { Monogenic } \\
\text { neonatal } \\
\text { diabetes } \\
(n=54)\end{array}$ & $\begin{array}{l}\text { Disease of } \\
\text { exocrine } \\
\text { pancreas }^{3} \\
(n=59)\end{array}$ & $\begin{array}{l}\text { Drug- or } \\
\text { chemical- } \\
\text { induced } \\
\text { diabetes } \\
(n=48)\end{array}$ & $\begin{array}{l}\text { Diabetes } \\
\text { associated with a } \\
\text { genetic } \\
\text { syndrome } \\
(n=65)\end{array}$ \\
\hline Gender male/female (\%) & $54 / 46$ & $35 / 65$ & $50 / 50$ & $41 / 59$ & $42 / 58$ & $40 / 60$ & $58 / 42$ \\
\hline Age at diagnosis (y) & $7.9(4.5 ; 11.8)$ & $11.7(8.5 ; 14.0)$ & $11.0(4.9 ; 13.5)$ & $0.2(0.0 ; 0.5)$ & $14.1(10.8 ; 16.4)$ & $12.1(9.1 ; 15.1)$ & $7.3(2.4 ; 11.0)$ \\
\hline Diabetes duration (y) & $3.9(2.0 ; 6.6)$ & $5.1(2.5 ; 7.1)$ & $1.7(0.6 ; 4.4)$ & $5.2(2.2 ; 11.0)$ & $5.0(1.9 ; 10.4)$ & $2.7(0.5 ; 4.8)$ & $8.3(2.8 ; 11.5)$ \\
\hline Daily insulin dose (U/kg) & $0.2(0.1 ; 0.5)$ & $0.6(0.3 ; 0.8)$ & $0.7(0.6 ; 0,9)$ & $0.7(0.5 ; 0.8)$ & $0.7(0.5 ; 1.0)$ & $0.8(0.4 ; 1.2)$ & $1.0(0.7 ; 1.2)$ \\
\hline $\begin{array}{l}\text { Oral antihyperglycemic } \\
\text { agents (\%) }\end{array}$ & 2 & 15 & 18 & 17 & 5 & 8 & 9 \\
\hline $\mathrm{A} 1 \mathrm{C}(\%)$ & $6.2(6.0 ; 6.4)$ & $6.7(5.9 ; 7.6)$ & $6.2(5.7 ; 6.7)$ & $6.6(5.7 ; 7.4)$ & $6.6(5.6 ; 7.4)$ & $6.6(6.2 ; 8.3)$ & $7.2(6.5 ; 8.2)$ \\
\hline
\end{tabular}

MODY, maturity onset diabetes of the young.

${ }^{1}$ Data presented as median (lower quartile; upper quartile) or percentage.

${ }^{2}$ Owing to small group size, no statistical comparison is presented.

${ }^{3}$ Excludes cystic fibrosis-related diabetes.

TABLE 3 Comparison between type 1 diabetes, type 2 diabetes, and CFRD ${ }^{1}$

\begin{tabular}{|c|c|c|c|}
\hline & T1D $(n=25889)$ & T2D $(n=347)$ & CFRD $(n=193)$ \\
\hline Gender male/female (\%) & $52 / 48$ & $40 / 60^{2}$ & $44 / 56^{4}$ \\
\hline Age at diagnosis (years) & $7.8(4.3 ; 11.2)$ & $13.8(12.0 ; 15.5)^{2}$ & $12.9(10.8 ; 14.8)^{2,3}$ \\
\hline Documented insulin treatment (\%) & & $27^{2}$ & $57^{2,3}$ \\
\hline Daily insulin dose $(\mathrm{U} / \mathrm{kg})$ & $0.9(0.7 ; 1.1)$ & $0.7(0.3 ; 0.9)^{2}$ & $0.4(0.1 ; 0.8)^{2,3}$ \\
\hline $\mathrm{A} 1 \mathrm{C}(\%)$ & $7.9(7.1 ; 8.9)$ & $7.1(6.0 ; 8.9)^{2}$ & $6.0(5.6 ; 6.7)^{2,3}$ \\
\hline BMI z-score & $0.5(-0.2 ; 1.2)$ & $2.5(1.8 ; 3.2)^{2}$ & $-0.5(-1.00 .1)^{2,3}$ \\
\hline
\end{tabular}

CFRD, cystic fibrosis related diabetes; T1D, type 1 diabetes; T2D, type 2 diabetes.

${ }^{1}$ Data presented as median (lower quartile; upper quartile) or percentages.

${ }^{2}$ Indicates a statistical difference with T1D with a $P$-value $<.01$.

${ }^{3}$ Indicates a statistical difference with T2D with a $P$-value $\leq .01$.

${ }^{4}$ Indicates a statistical difference with T1D with a $P$-value $=.03$.

of diabetes ranged from $0 \%$ to $9.6 \%$. There is also a weak correlation between center size and the percentage of patients with other forms included in their SWEET cohort (Spearman Rho 0.38, $P=.007$ ).

In preparation for this manuscript, we asked each center where would patients with T2D, cystic fibrosis, and diabetes in pregnancy in youth (gestational or pre-existing) be followed (Table 4). Interestingly most centers indicated that for both, T2D and CFRD, they would be involved in the follow up either within their pediatric diabetes clinic or by the pediatric diabetes team within a joint clinic. For diabetes in pregnancy (either gestational diabetes or pre-existing T1D) answers were more variable with over half of the centers transferring care to a diabetes pregnancy clinic.

\section{4 | DISCUSSION}

This report provides a clinical description of other forms of diabetes encountered in the SWEET database, reflecting real world clinical practice of a large, multinational consortium of centers caring for children with diabetes. Both observed frequency and clinical characteristics of non-T1D in this database are similar to published literature.

The frequency of non-T1D $(<5 \%)$ remains a small proportion of children followed by participating clinics. Although we cannot measure prevalence or incidence through the SWEET database, it is interesting that the distribution of the different forms of diabetes within the database is in line with most reports of prevalence or incidence for T2D and other forms of diabetes, which are between $2 \%$ and $15 \%{ }^{2,3}$ When using similar clinical criteria applied by expert clinicians, the proportion of children with $\mathrm{T} 2 \mathrm{D}$ has been reported to be as high as $11 \%$ of all children with diabetes in the USA, ${ }^{4}$ whereas it remains a much lower proportion in Europe..$^{2,5,11,12}$ Contrary to North American reports, ${ }^{4,13}$ the frequency of T2D is usually similar to that of MODY in European countries. ${ }^{11,14}$ At least two European centers have published higher frequency of MODY than T2D. ${ }^{2,5}$ The differential diagnosis between MODY and T2D or T1D remains clinically difficult and requires genetic testing. ${ }^{15}$ Examples from the literature 
TABLE 4 Center survey responses on where children and adolescents with type 2 diabetes CFRD, and adolescents with diabetes in pregnancy are followed ${ }^{1}$

\begin{tabular}{|c|c|c|c|c|}
\hline & $\begin{array}{l}\text { Within pediatric } \\
\text { diabetes }\end{array}$ & $\begin{array}{l}\text { Within a disease- } \\
\text { specific clinic }{ }^{2}\end{array}$ & $\begin{array}{l}\text { No clear } \\
\text { policy }\end{array}$ & $\begin{array}{l}\text { Joint clinic for CFRD } \\
\text { and diabetes } \\
3^{3}\end{array}$ \\
\hline CFRD & $3(9)$ & $3(9)$ & 1 (3) & $23(72)$ \\
\hline Known patient with type 1 diabetes who becomes pregnant & $9(28)$ & $15(47)$ & $7(22)$ & \\
\hline New adolescent patient with gestational diabetes & $8(25)$ & $17(53)$ & $7(22)$ & \\
\hline
\end{tabular}

CRDR, cystic fibrosis-related diabetes.

${ }^{1}$ Data are presented as number (percentages) of respondents.

${ }^{2}$ Within each question this was given as obesity clinic for type 2 diabetes, CFRD clinic for CFRD and diabetes in pregnancy clinic for the two questions on pregnancy.

${ }^{3}$ The option of a joint clinic was offered for CFRD only.

suggest that centers with higher frequency of MODY as compared with T2D had easy access to genetic testing resulting in more systematic testing of children with negative antibody regardless of clinical characteristics. ${ }^{2,5,13}$ Because the majority of SWEET centers are located in Europe, it is not surprising that the frequencies seen here are similar to those reports. As SWEET continues to grow and more centers from outside Europe join, it will be interesting to monitor if these proportions change.

Use of the ISPAD classification of diabetes ${ }^{8}$ to document diabetes type is a sound starting point. However, as seen within this project, in some cases it remains difficult to assign a diabetes subtype. Some centers may not have access to genetic testing. Other children may have been tested but found to be negative for the known genetic causes. In other cases, there is no clear category to choose from. For example, an infant with pancreatic agenesis with negative genetic testing could be classified under monogenic forms of diabetes (as this remains most likely) or under exocrine pancreatic disease. Some diagnoses may overlap between categories such as diabetes secondary to pancreatitis caused by chemotherapy, which could be entered under pancreatitis or under drug or chemical-induced diabetes. New specific drugs causing diabetes, such as atypical psychotic agents and calcineurin inhibitors, have emerged since the start of SWEET and are not listed in the current list. When dealing with large databases, it is impractical to verify each case for clarification and consistency. Although, the list of other forms of diabetes designed for SWEET in 2006 was carefully thought out and used the published ISPAD classification available at the time, new etiologic diagnoses have become available over the last decade. This suggests that the current SWEET classification should be revised at regular intervals to ensure inclusion of new diagnostic entities. Many of the other forms of diabetes require genetic testing for confirmation of the diagnosis. Being aware of the high number of children with unclassified subtype and unclassified MODY, collaboration with the ISPAD rare forms of diabetes center in Exeter (http://www. diabetesgenes.org/content/information-known-types-rare-diabetes) and EURO-WABB project (http://web.ispad.org/resource-type/ispadrare-diabetes) will also allow SWEET centers without genetic facilities to classify their cases appropriately. Lastly, individual centers also need to be attentive and update the diagnosis for each case when more information becomes available over time.

Another difficulty that arises with this classification for research is the wide variety of pathologies that are included in the same subgroup. For example, analyzing outcome of genetic defect of $\beta$-cell function as a single group does not provide us with a clear clinical picture as it includes neonatal diabetes which often requires early and lifelong treatment, with MODY 2 which has a benign course and other MODY which may be associated with early complications. The same issue is present within most of the other larger categories of this classification. Hence, when presenting clinical outcome, these etiologic classification categories need to be subdivided in order to provide more accurate and pertinent information.

The proportion of children with MODY 2 being on insulin is worth discussing. A previous report from the DVP initiative had observed a similar rate of insulin use (8\%) in pediatric patients with MODY $2 .{ }^{12}$ This form of diabetes is associated with mild hyperglycemia often present from birth ${ }^{16}$ and slight increase in A1C with slight deterioration with age similar to what is seen in individuals without diabetes. ${ }^{17,18}$ Pharmaceutical treatment does not normalize glycemia. ${ }^{19}$ Further, this condition is rarely associated with the typical long-term complications of diabetes even in the absence of treatment. ${ }^{6}$ Between the monetary cost, personal burden associated with daily insulin injections and potential risk of hypoglycemia, it seems surprising to have $7 \%$ of children with MODY 2 on this treatment. Nevertheless, it was reassuring to see the total daily dose being minimal, probably resulting in minimal risk of hypoglycemia. Further inquiry of those cases could help us understand the reasons behind the ongoing use of insulin.

In the survey, most centers expected to be following the majority of children with T2D within their catchment area. Without population base data, it is difficult to ascertain the validity of this information. However, both Amed et al. ${ }^{13}$ and Neu et al. ${ }^{11}$ found that over $90 \%$ of the children with T2D and rare forms of diabetes in their study were being followed by pediatric diabetes specialists or pediatricians. Nevertheless, it is possible that children with T2D are underrepresented in this database because they are followed elsewhere unbeknownst to the pediatric diabetes clinics in SWEET, or because they remain undiagnosed. ${ }^{20}$ The lower frequency could also be related to lower frequency of childhood obesity. ${ }^{1}$ Another point to consider for children with T2D is that most pediatric diabetes programs are built around the clinical management of children with T1D. Even if some aspects of diabetes care overlap, the needs for managing T2D may be different from those for managing T1D. For example, the management of $T 2 D$ requires intensive lifestyle changes often 


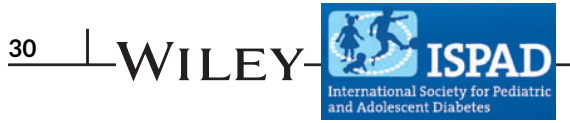

coupled with significant psychosocial interventions ${ }^{21}$ that may be better served in a pediatric obesity clinic with collaboration with pediatric diabetes specialists. However, pediatric obesity clinics are not always available and the pediatric diabetes clinic then needs to adapt its management for those children. It remains to be proven which type of clinic would serve this population best. The SWEET collaboration offers an avenue to discuss these adaptations to meet the clinical needs of T2D.

The group of children with CFRD in the SWEET database was similar to the literature in terms of age of onset, weight and use of insulin. ${ }^{22,23}$ Despite clear recommendation to initiate insulin early for better overall outcome, ${ }^{24}$ there is often resistance to initiate this treatment from the families and care givers resulting in one in five children with CFRD not being on insulin. ${ }^{25}$ When organizing diabetes care for children with CFRD, one must consider amongst other issues, a dietary approach focused on high energy demands ${ }^{24}$ and infection control measures. ${ }^{26}$ Close collaboration with the cystic fibrosis clinic is required. Accordingly, 23 out of 32 clinics in the survey stated doing joint follow-up with cystic fibrosis teams for these patients, which is a higher proportion than in the UK. ${ }^{27}$

When considering the other causes of diabetes in children, most pediatric diabetologists are expected to be involved in the care of a few individuals with special rare forms of diabetes throughout their career. The weak correlation between center size and the percentage of other forms of diabetes in each participating center can be explained in part by the numbers (ie, by random effect, larger centers will be more likely to see rare forms). It could also be explained by a referral bias by which unusual forms of diseases are more likely to be sent to larger, more specialized clinics. Despite this correlation, each center remained with very few cases of each specific etiology. Being able to combine these cases in the SWEET database allows reporting on a significant number of patients with similar rare etiology that could not be gathered otherwise. Beyond this publication, the SWEET structure allows for better descriptions through internal reports and case discussion at the annual meeting. Moreover, discussion of organization of care through the peer review process may help pediatric diabetes specialists to provide a more targeted approach to these rare forms of diabetes.

This report has several limitations. First, this database is not population based. Therefore, no prevalence or incidence rates can be deduced. Further, it is based on reported coding according to physician's opinion. There is no systematic collection of information on initial diagnosis, laboratory or genetic testing to support the diagnosis. Within this database, it is not possible to distinguish between suspected or proven cases and, for those labeled as undetermined, between those not investigated versus those thoroughly investigated but without a specific diagnosis. Coding may not be consistent throughout centers or even within centers. However, the size of the population reported is unique and allows overcoming some of the limits listed above.

In conclusion, forms of diabetes other than type 1 in the SWEET dataset remain rare and at times difficult to characterize. Because each center has only a few cases of rare forms of diabetes, the SWEET collaboration allows pediatric diabetes centers to share their experiences resulting in increased awareness, knowledge, and improve patient care. As our knowledge evolves, it will be important to continue monitoring of the frequency and clinical outcomes of the different rare forms of diabetes in this database.

\section{ACKNOWLEDGMENTS}

We are thankful for the following individuals for their support of this work: Katarina Fink for data management support, Andreas Hungele and Ramona Ranz for DPV documentation software support, Michael Witsch for center integration, Thomas Danne and Olga Kordonori for initiating the SWEET collaboration. We would also like to recognize all patients and their families who participate in SWEET and the staff of participating centers. Without them, the SWEET collaboration would not be possible.

\section{Funding information}

We would like to thank AstraZenaca for funding this special issue of Pediatric Diabetes. The work of SWEET is possible through the support of the following sponsors: AstraZeneca, Boehringer Ingelheim, DexCom Inc., Diabetes Foundation UK, Foundation Hannoversche Kinderheilanstalt, Lilly Diabetes Excellence Centre, Medtronic Europe, the Medtronic Foundation, and Sanofi.

\section{Conflict of interest}

We declare that we have no conflict of interest.

\section{Author contributions}

D. P. wrote the manuscript. A. S., C. de B., K. C., J. B., N. H. B., M. C., N. B., C. L., S. O., R. R., A. G. V., L. P., R. V., I. D. K. researched data und reviewed/edited the manuscript. A. S. analyzed the data. All coauthors approved the final version to be published.

How to cite this article: Pacaud D, Schwandt A, de Beaufort C, Casteels K, Beltrand J, Birkebaek $\mathrm{NH}$, Campagnoli M, Bratina N, Limbert C, O'Riordan S, Ribeiro R, Gerasimidi-Vazeou A, Petruzelkova L, Verkauskiene R, Krisane ID, the SWEET Study Group. A description of clinician reported diagnosis of type 2 diabetes and other non-type 1 diabetes included in a large international multicentered pediatric diabetes registry (SWEET), Pediatr Diabetes 2016, 17 (Suppl. 23), 24-31. DOI:10.1111/pedi.12426

\section{REFERENCES}

1. Patterson C, Guariguata L, Dahlquist G, Soltész G, Ogle G, Silink M. Diabetes in the young-a global view and worldwide estimates of numbers of children with type 1 diabetes. Diabetes Res Clin Pract. 2014;103:161-175.

2. Mozzillo E, Salzano G, Barbetti F, et al. Survey on etiological diagnosis of diabetes in 1244 Italian diabetic children and adolescents: impact of access to genetic testing. Diabetes Res Clin Pract. 2015;107(3): e15-e18.

3. Rubio-Cabezas O, Hattersley AT, Njolstad PR, et al., ISPAD Clinical Practice Consensus Guidelines 2014. The diagnosis and management 
of monogenic diabetes in children and adolescents. Pediatr Diabetes. 2014;15(Suppl. 20):47-64.

4. Pettitt DJ, Talton J, Dabelea D, et al. Prevalence of diabetes in U.S. youth in 2009: the SEARCH for diabetes in youth study. Diabetes Care. 2014;37(2):402-408.

5. Fendler W, Borowiec M, Baranowska-Jazwiecka A, et al. Prevalence of monogenic diabetes amongst Polish children after a nationwide genetic screening campaign. Diabetologia. 2012;55(10):2631-2635.

6. Chakera AJ, Steele AM, Gloyn AL, et al. Recognition and management of individuals with hyperglycemia because of a heterozygous glucokinase mutation. Diabetes Care. 2015;38(7):1383-1392.

7. Danne T, Lion S, Madaczy L, et al. Criteria for centers of reference for pediatric diabetes-a European perspective. Pediatr Diabetes. 2012;13(Suppl. 16):62-75.

8. Craig ME, Jefferies C, Dabelea D, et al., ISPAD Clinical Practice Consensus Guidelines 2014. Definition, epidemiology, and classification of diabetes in children and adolescents. Pediatr Diabetes. 2014;15(Suppl 20):4-17.

9. World Health Organization. WHO growth reference 5-19 years: BMI-for-age (5-19 years). http://www.who.int/growthref/who2007_ bmi_for_age/en/index.html. Accessed 29 April, 2016.

10. American Diabetes Association, European Association for the Study of Diabetes, International Federation of Clinical Chemistry Laboratory Medicine, and International Diabetes Federation. Consensus statement on the worldwide standardisation of the HbA1c measurement. Diabetologia. 2007;50(10):2042-2043.

11. Feldhahn L, Dietz K, Willasch AM, Neu A; DIARY-Group BadenWuerttemberg. Type 2 diabetes mellitus in children and adolescents is still a rare disease in Germany: a population-based assessment of the prevalence of type 2 diabetes and MODY in patients aged 0-20 years. Pediatr Diabetes. 2009;10(7):468-473.

12. Schober E, Rami B, Grabert M, et al. Phenotypical aspects of maturity-onset diabetes of the young (MODY diabetes) in comparison with type 2 diabetes mellitus (T2DM) in children and adolescents: experience from a large multicentre database. Diabet Med. 2009;26(5):466-473.

13. Amed S, Hamilton JK, Sellers EA, et al. Differing clinical features in Aboriginal vs. non-Aboriginal children presenting with type 2 diabetes. Pediatr Diabetes. 2012;13(6):470-475.

14. Irgens HU, Molnes J, Johansson BB, et al. Prevalence of monogenic diabetes in the population-based Norwegian Childhood Diabetes Registry. Diabetologia. 2013;56(7):1512-1519.
15. Shields BM, Hicks S, Shepherd MH, Colclough K, Hattersley AT, Ellard S. Maturity-onset diabetes of the young (MODY): how many cases are we missing? Diabetologia. 2010;53(12):2504-2508.

16. Prisco F, lafusco D, Franzese A, Sulli N, Barbetti F. MODY 2 presenting as neonatal hyperglycaemia: a need to reshape the definition of "neonatal diabetes"? Diabetologia. 2000;43(10):1331-1332.

17. Pearson ER, Starkey BJ, Powell RJ, Gribble FM, Clark PM, Hattersley AT. Genetic cause of hyperglycaemia and response to treatment in diabetes. Lancet. 2003;362(9392):1275-1281.

18. Steele AM, Shields BM, Wensley KJ, Colclough K, Ellard S, Hattersley AT. Prevalence of vascular complications among patients with glucokinase mutations and prolonged, mild hyperglycemia. JAMA. 2014;311(3):279-286.

19. Stride A, Shields B, Gill-Carey O, et al. Cross-sectional and longitudinal studies suggest pharmacological treatment used in patients with glucokinase mutations does not alter glycaemia. Diabetologia. 2014;57(1):54-56.

20. Jackson-Leach R, Lobstein T. Estimated burden of paediatric obesity and co-morbidities in Europe. Part 1. The increase in the prevalence of child obesity in Europe is itself increasing. Int $J$ Pediatr Obes. 2006;1(1):26-32.

21. Zeitler P, Fu J, Tandon N, et al., ISPAD Clinical Practice Consensus Guidelines 2014. Type 2 diabetes in the child and adolescent. Pediatr Diabetes. 2014;15(Suppl. 20):26-46.

22. Ode KL, Moran A. New insights into cystic fibrosis-related diabetes in children. Lancet Diabetes Endocrinol. 2013;1(1):52-58.

23. Scheuing N, Holl RW, Dockter G, et al. Diabetes in cystic fibrosis: multicenter screening results based on current guidelines. PLoS One. 2013;8(12):e81545.

24. Moran A, Pillay K, Becker DJ, Acerini CL, International Society for Pediatric and Adolescent Diabetes. ISPAD Clinical Practice Consensus Guidelines 2014. Management of cystic fibrosis-related diabetes in children and adolescents. Pediatr Diabetes. 2014;15(Suppl 20):65-76.

25. Scheuing N, Berger G, Bergis D, et al. Adherence to clinical care guidelines for cystic fibrosis-related diabetes in 659 German/Austrian patients. J Cyst Fibros. 2014;13(6):730-736.

26. Saiman L, Siegel JD, LiPuma JJ, et al. Infection prevention and control guideline for cystic fibrosis: 2013 update. Infect Control Hosp Epidemiol. 2014;35(Suppl. 1):S1-S67.

27. Wickens-Mitchell KL, Gilchrist FJ, McKenna D, Raffeeq P, Lenney W. The screening and diagnosis of cystic fibrosis-related diabetes in the United Kingdom. J Cyst Fibros. 2014;13(5):589-592. 